\title{
Validación Española del "Learning Climate Questionaire" Adaptado a la Educación Física
}

\author{
Spanish Version of the Learning Climate Questionnaire Adapted \\ to Physical Education
}

\author{
Antonio Granero-Gallegos*, ${ }^{*}$, Antonio Baena-Extremera ${ }^{b}$ José Antonio Sánchez-Fuentes $^{b}$ \\ \& Marina Martínez -Molina ${ }^{b}$ \\ ${ }^{a}$ Consejería de Educación de la Junta de Andalucía, Sevilla, España \\ $\&^{b}$ Universidad de Murcia, Murcia, España
}

\begin{abstract}
Resumen
El objetivo de esta investigación fue analizar las propiedades psicométricas del Learning Climate Questionnaire (LCQ) adaptado a la Educación Física (EF) en una muestra española. Se pretende determinar si es un instrumento válido y fiable para su utilización en futuras investigaciones. Se administró a un total de 758 estudiantes de secundaria de 12 a 18 años, analizándose la estructura del instrumento mediante procedimientos confirmatorios. Los análisis apoyan el modelo teórico hipotetizado de un factor (apoyo a la autonomía). La versión española del instrumento para EF mostró niveles aceptables de consistencia interna y estabilidad temporal. La validez concurrente se exploró examinando la relación de la escala estudiada con los distintos tipos de motivación autodeterminada, mediante modelos de ecuaciones estructurales.
\end{abstract}

Palabras clave: Educación Física, apoyo a la autonomía, propiedades psicométricas, LCQ-EF.

\begin{abstract}
The objective of this research was to analyze the psychometric properties of the Learning Climate Questionnaire (LCQ) adapted to Physical Education (PE) in a Spanish sample. The purpose is to determine whether it is a valid and reliable tool to be used in future research._It was administered to a total of 758 high school students from 12 to 18 years old, analyzing the structure of the instrument using confirmatory procedures. Analyses support the hypothesized theoretical model of a specific factor, the autonomy support. The Spanish version of the instrument for PE showed acceptable levels of internal consistency and temporal stability. Concurrent validity was explored by examining the relationship of the dimension studied with different types of self-determined motivation, using structural equation modeling.

Keywords: Physical Education, autonomy support, psychometric properties, LCQ-PE.
\end{abstract}

Para que un sistema educativo sea exitoso requiere alumnado motivado; pero también es importante entender que estos estudiantes requieren profesores que motiven, pues de ellos dependerá que se pueda crear un ambiente adecuado para el aprendizaje (Boekaerts, 1996). El docente es uno de los principales agentes encargados de crear un clima acorde para el aprendizaje del alumnado. Estudios como el de Standage, Duda y Ntoumanis, (2005) ponen de manifiesto la importancia de la motivación en los alumnos de cara a su aprendizaje, teniendo como referente la Teoría de la Autodeterminación (Deci \& Ryan, 1985; Ryan \& Deci, 2000). Esta Teoría se basa en el supuesto de que las personas están intrínsecamente motivados hacia el aprendizaje, el crecimiento e, incluso, el desafío intelectual.

* Dirección postal: Centro del Profesorado Almería 03, Ctra. De Águila s/n, Cuevas del Almanzora, Almería,

España 04610. E-mail: agranerog@hotmail.com
Siguiendo las directrices básicas de esta teoría aplicadas al ámbito escolar, se destaca que en un centro educativo los discentes actuarían motivados por tres posibles necesidades (Deci \& Ryan, 1985): necesidad de competencia, necesidad de autonomía o necesidad de relacionarse.

Centrándonos en la autonomía, ésta se refiere a la necesidad de experimentarse como iniciador y regulador de las acciones que uno mismo realiza. De esta forma, Williams, Saizow, Ross y Deci (1997) hablan del apoyo a la autonomía como el grado en el que profesores reconocen la capacidad del alumnado y fomentan su participación activa en actividades de aprendizaje. El apoyo a la autonomía supone un proceso activo por parte de los docentes que facilitaría, según estos autores, la participación de los discentes en su aprendizaje autodeterminado. De esta forma, cuando un profesor crea un clima de apoyo a la autonomía, los estudiantes se sienten escuchados y comprendidos, experimentando libertad para actuar. Ello contribuye a 
que terminen sintiéndose bien consigo mismos y mejoren la toma de decisiones en clase.

Teniendo en cuenta lo anterior, la motivación de un alumno en Educación Física (EF) dependerá de diversos factores, entre los que destaca la autonomía. A nivel educativo, y enlazando nuevamente con la Teoría de la Autodeterminación, se predice que los climas de aprendizaje que apoyan a la autonomía de los estudiantes mejorarán su motivación intrínseca (Black \& Deci, 2000; Reeve, Jang, Carrell, Jeon, \& Barsh, 2004), mientras que los climas en los que los estudiantes perciben un control de su comportamiento, disminuye su sentido de la voluntad y se reduce la motivación natural. A esto hay que sumarle que, según Matos (2009), la motivación intrínseca es el mejor ejemplo del comportamiento autónomo y autodeterminado. Por contra, la motivación extrínseca es más controlada y menos autónoma. Se puede concretar que es importante promover el apoyo a la autonomía porque esta predice la autodeterminación; y en diversos trabajos se ha demostrado que está vinculada con una mayor concentración en clase (Black \& Deci, 2000; Standage et al., 2005), un mayor esfuerzo académico (Ntoumanis, 2001) y un menor abandono escolar (Black \& Deci, 2000).

Para estudiar la autonomía, Williams y Deci (1996) crearon el Learning Climate Questionnaire (LCQ). Este instrumento fue adatado al deporte, en España, por Balaguer, Castillo, Duda y Tomás (2009), quienes desarrollaron el Cuestionario de Clima en el Deporte. Esta escala consta, al igual que la original, de 15 ítems en su versión completa. En ella se evalúa la percepción que tienen los deportistas del grado de apoyo a la autonomía ofrecido por su entrenador. Siguiendo a Balaguer et al. (2009), las medidas recogidas con este instrumento pueden ser utilizadas para poner a prueba los modelos teóricos que se postulan desde la Teoría de la Autodeterminación, así como para comprobar empíricamente si el clima que genera el profesor, en el caso de la $\mathrm{EF}$, produce algún tipo de efecto o consecuencias sobre determinadas variables del alumnado con quien interacciona.

En relación a la EF existen aún muy pocos trabajos con el LCQ, entre los que se encuentran los de Hagger, Chatzisarantis, Culverhouse y Biddle, (2003) y Ntoumanis (2005). A pesar de la importancia de dicha escala para el contexto educativo (Baena-Extremera, Granero-Gallegos, Sánchez-Fuentes, \& Martínez-Molina, 2013), aún no existe una versión específica para EF en español. Teniendo en cuenta lo expuesto, y según las indicaciones de CarreteroDios y Pérez (2007), en este trabajo se presentan los resultados de dos estudios independientes y se expone el proceso de traducción y adaptación de los ítems, así como los datos relacionados con las evidencias de validez de contenido de los mismos.

\section{Estudio 1: Estudio Psicométrico Preliminar del LCQ}

\section{Método}

\section{Participantes}

En este primer estudio participaron un total de 300 alumnos $(140$ varones $=46.6 \% ; 160$ mujeres $=53.4 \%)$ de tres centros secundaria de la provincia de Almería. Con ello, se supera ampliamente el número mínimo de sujetos para el estudio piloto de una escala (Osterlind, 1989). El rango de edad estuvo comprendido entre 12 y 18 años $(M=15.51 ; S D=1.31)$, siendo la edad media de los chicos $15.56(S D=1.34)$, y la de las chicas $15.46(S D=1.29)$.

\section{Instrumento}

Se adaptó la versión original del LCQ de Williams y Deci (1996), basada en el Health-Care Climate Questionnaire (Williams, Grow, Freedman, Ryan, \& Deci, 1996), que consta de 15 ítems para medir el apoyo a la autonomía por parte del profesor mediante una dimensión: apoyo a la autonomía. En las instrucciones se pide que indiquen el grado de acuerdo con los ítems, recogiéndose las respuestas en una escala de ítems politómicos de 7 puntos que oscila desde Muy en desacuerdo (1) a Muy de acuerdo (7).

Traducción y Fases Previas del Proceso de Adaptación. La adaptación española del LCQ se realizó atendiendo a los estándares metodológicos internacionales recomendados por la International Test Comision para adaptar correctamente test y escalas de unas culturas a otras (Muñiz, 2000; Muñiz \& Bartram, 2007). Para evitar imprecisiones, se combinaron los diseños de traducción directa e inversa de los ítems, según el procedimiento parallel back translation (Brislin, 1986).

La evaluación cualitativa de ítems (validez de contenido) se efectuó mediante el juicio de cuatro expertos (Osterlind, 1989): dos en construcción de escalas y dos conocedores del constructo a evaluar. Todos los ítems fueron analizados y revisados hasta que se recogiese la dimensión teórica de la forma más clara y precisa. El encabezamiento fue: "Piensa en tu profesor/a de Educación Física y cómo él o ella interactúa contigo durante la clase".

La nueva versión fue administrada a 50 alumnos de secundaria con edades entre 12 y 18 años. Los comentarios sobre instrucciones y forma de redacción supusieron cambios menores. Tras el análisis de los resultados psicométricos obtenidos, y una última revisión por el equipo de investigación, se llegó a la versión final del LCQ adaptado a la EF (LCQ-EF).

\section{Procedimiento}

Se obtuvo permiso de los órganos de dirección de los centros educativos para realizar la investigación. Los estudiantes fueron informados del propósito del estudio y 
Granero-Gallegos, A., Baena-Extremera, A., Sánchez-Fuentes, J. A. \& Martínez-Molina, M. (2014). Validación Española del "Learning Climate Questionaire" Adaptado a la Educación Física.

de sus derechos como participantes en el mismo; asimismo, este trabajo posee informe favorable de la Comisión de Bioética. Los instrumentos para medir las diferentes variables se administraron en el aula sin la presencia del docente. Cada participante tuvo 10-20 minutos para completar los cuestionarios. Las respuestas se mantendrán en el anonimato.

\section{Análisis de Datos}

Para alcanzar el primer objetivo de este trabajo, en primer lugar se realizó un análisis factorial exploratorio (EFA) para obtener la estructura factorial interna de la escala y se analizó la consistencia interna para determinar la fiabilidad (alfa) de Cronbach de la dimensión. Se llevó a cabo un análisis de ítems y homogeneidad de la escala con el objeto de analizar métricamente las propiedades de cada reactivo y valorar cada uno de ellos desde el punto de vista estadístico. Para estos análisis se utilizó uno de los paquetes estadísticos más utilizados en cien- cias sociales, el SPSS (Statistical Package for the Social Sciences) v. 17.0

\section{Resultados}

\section{Análisis de la Estructura Interna}

Se llevó a cabo un EFA utilizando el método de extracción de componentes principales (PCA) requiriéndose una correlación mínima de .40 para que cada ítem fuese importante dentro del factor (Stevens, 1992). La medida de adecuación muestral de Kaiser-Meyer-Olkin (KMO) es aceptable y la prueba de esfericidad de Bartlett resultó estadísticamente significativa, lo que permitió concluir la pertinencia de la aplicación del EFA. Con un porcentaje total de varianza explicada del $63.98 \%$, los resultados confirman la estructura dimensional en un solo factor con saturaciones de los ítems por encima del .72, a excepción del ítem 13 (-.15) que no alcanza el coeficiente de visualización de .40 (Tabla 1).

Tabla 1

Estructura Factorial, Comunalidades, Autovalores, Alfa de Cronbach y Porcentaje de Varianza Explicada (N=300)

Factor

Apoyo autonomía

2 Me siento comprendido/a por mi profesor/a de EF .89

4 Mi profesor/a de EF me transmite confianza en mi habilidad para hacerlo bien en las clases

8 Confío mucho en mi profesor/a de EF

11 Mi profesor/a de EF maneja muy bien las emociones de la gente

9 Mi profesor/a de EF responde a mis preguntas con esmero (detenidamente y en detalle)

5 Me siento aceptado/a por mi profesor/a de EF

$10 \mathrm{~A}$ mi profesor/a de EF le interesa saber como me gusta hacer las cosas

7 Mi profesor/a de EF me anima a que pregunte en clase las dudas que tenga

3 Tengo la posibilidad de poder comunicarme fácilmente con mi profesor/a durante la clase de EF

6 Mi profesor/a de EF se asegura que yo realmente entienda cuál es el objetivo de cada actividad de clase y de lo que tengo que hacer

12 Siento que mi profesor/a de EF se preocupa de mí como persona

14 Mi profesor/a de EF trata de entender como veo las cosas antes de sugerirme una nueva

1 Siento que mi profesor/a de EF me ofrece distintas alternativas y opciones

Autovalor

Varianza explicada

Nota. Coeficiente de visualización .40; $h^{2}$ : comunalidad. 
Análisis de Ítems y Homogeneidad de la Escala

En el análisis estadístico de ítems se mantuvo la distribución ítem-factor observada en el instrumento original (Williams \& Deci, 1996). Los criterios que se tuvieron en cuenta para conservar un ítem fueron: coeficiente de correlación corregido ítem-total (CCIT-c) $\geq .30$, desviación típica $(S D)>1$, y todas las opciones de respuesta usadas en algún momento (Nunnally \& Bernstein, 1995). Los índices de asimetría y curtosis son próximos a 0 y $<2$ (Tabla 2). Los ítems del factor apoyo a la autonomía presentaron valores medios entre 5.13 para el ítem 5 y 3.08 para el ítem 13. Las $S D$ fueron $>1$. La consistencia interna fue adecuada ( $\alpha=.95)$. Todos los CCIT-c presentaron valores >.71, excepto el ítem 13 (-.12).

Autores como Carretero-Dios y Pérez (2007) recomiendan realizar un estudio de correlación para asegurar la homogeneidad de cada dimensión (CC). En el presente trabajo, la correlación entre la puntuación de cada ítem y la puntuación total en cada uno de los componentes muestran correlaciones positivas con la dimensión teórica a la que pertenecen (apoyo a la autonomía), excepto el ítem 13 ( $r=-.03)$; asimismo, el CCIT-c es $<.30$ y esto, según Nunnally y Bernstein (1995), supone que ese ítem debería ser eliminado de la escala.

Tabla 2

Estadisticos Descriptivos, de Consistencia Interna y de Homogeneidad ( $N=300)$

\begin{tabular}{lccccccc}
\hline Escala: & $M$ & $S D$ & CCIT-c & CC & $\alpha$ sin ítem & Asimetría & Curtosis \\
\hline Apoyo a la autonomía $(\alpha=.95)$ & & & & & & & \\
Ítem-2 & 4.72 & 1.96 & .85 & .88 & .94 & -.49 & -.88 \\
Ítem-4 & 4.87 & 1.86 & .83 & .86 & .94 & -.48 & -.85 \\
Ítem-8 & 4.86 & 1.92 & .83 & .86 & .94 & -.48 & -.95 \\
Ítem-11 & 4.45 & 1.94 & .83 & .86 & .94 & -.36 & -.95 \\
Ítem-9 & 4.78 & 1.86 & .80 & .83 & .94 & -.47 & -.82 \\
Ítem-5 & 5.13 & 1.80 & .80 & .82 & .94 & -.65 & -.59 \\
Ítem-10 & 4.29 & 1.93 & .82 & .85 & .94 & -.28 & -1.01 \\
Ítem-7 & 4.79 & 1.87 & .78 & .81 & .94 & -.47 & -.87 \\
Ítem-3 & 4.96 & 1.87 & .80 & .83 & .94 & -.62 & -.68 \\
Ítem-6 & 4.91 & 1.87 & .75 & .79 & .94 & -.50 & -.91 \\
Ítem-12 & 4.31 & 1.98 & .77 & .81 & .94 & -.20 & -1.12 \\
Ítem-14 & 4.33 & 1.86 & .75 & .78 & .94 & -.14 & -.88 \\
Ítem-1 & 4.41 & 1.80 & .72 & .76 & .94 & -.27 & -.76 \\
Ítem-15 & 3.85 & 2.12 & .72 & .77 & .94 & .04 & -1.31 \\
Ítem-13 & 3.08 & 2.12 & -.12 & -.03 & .96 & .56 & -1.10 \\
\hline
\end{tabular}

Nota. M: media; SD: desviación típica; CCIT-c: coeficiente de correlación corregido ítem-total; CC: correlación de cada ítem con la otra dimensión.

\section{Estudio 2: Análisis Confirmatorio del LCQ}

El objetivo de este trabajo es aportar evidencias sobre la dimensionalidad de la versión española del LCQ (LCQ-EF) en una muestra de adolescentes estudiantes de educación secundaria mediante procedimientos confirmatorios. Se analizaron las propiedades psicométricas de la versión española del LCQ adaptado a la EF; para ello se concretó: (a) examinar su estructura factorial con análisis factorial confirmatorio (AFC), (b) evaluar la consistencia interna mediante alfa de Cronbach, coeficiente de fiabilidad compuesta y varianza media extractada, (c) verificar su estabilidad temporal, y (d) evaluar la validez convergente del instrumento, analizando su relación con los distintos tipos de motivación autodeterminada mediante modelos de ecuaciones estructurales (SEM).
Método

\section{Participantes}

En este estudio participaron un total de 758 alumnos $(347$ hombres $=45.8 \% ; 411$ mujeres $=54.2 \%)$ de educación secundaria de la Región de Murcia. El rango de edad estuvo comprendido entre 12 y 18 años $(M=15.22 ; S D=1.27)$, siendo la edad media de los chicos $15.2(S D=1.29)$ y la de las chicas $15.18(S D=1.26)$. Para evaluar la estabilidad temporal se utilizó una segunda muestra de 150 alumnos, elegida previamente de forma aleatoria y compuesta por 73 chicos $(M=15.02 ; S D=1.37)$ y 77 chicas $(M=14.95$; $S D=1.42$ ) que completaron otra vez el instrumento siete semanas más tarde. 
Granero-Gallegos, A., Baena-Extremera, A., Sánchez-Fuentes, J. A. \& Martínez-Molina, M. (2014). Validación Española del "Learning Climate Questionaire" Adaptado a la Educación Física.

\section{Instrumentos}

Fue administrada la versión española del LCQ adaptado a EF, derivada del Estudio 1.

Escala de Motivación en el Deporte (SMS). Se empleó la versión validada al castellano por Núñez, Martín-Albo, Navarro y González (2006) y adaptada a EF. Consta de 28 ítems que miden los diferentes tipos de motivación establecidos por la Teoría de la Autodeterminación y que sugiere la explicación multidimensional de la motivación: desmotivación (4 ítems), motivación extrínseca (ME; 12 ítems; regulación externa, introyectada e identificada; 4 ítems para cada subescala), y motivación intrínseca (MI; 12 ítems). Las respuestas se recogieron en una escala de ítems politómicos con puntuación entre 1 (Totalmente en desacuerdo) y 7 (Totalmente de acuerdo). La consistencia interna hallada en este estudio estuvo entre $\alpha=.72$ para la $M E$ identificada y $\alpha=.91$ para la MI. El modelo con cinco dimensiones ha mostrado un satisfactorio índice de bondad de ajuste: $\chi^{2}=960.08, g l=340, p<.001, \chi^{2} / g l=2.82$, GFI $=.98$, $\mathrm{NFI}=.97, \mathrm{NNFI}=.98, \mathrm{CFI}=.98, \mathrm{RMSEA}=.05$.

\section{Procedimiento}

Se procedió de manera similar a la expuesta en el Estudio 1.

\section{Análisis Estadísticos}

La estructura factorial de la escala se evaluó mediante AFC con LISREL. Los análisis de ítems, homogeneidad y estructura interna, correlación y consistencia interna de la escala, se realizaron con el SPSS 17.0. La validez concurrente se evaluó mediante SEM con LISREL.

\section{Resultados}

\section{Descripción Inicial de los Datos}

En la Tabla 3 se muestran los estadísticos descriptivos del LCQ-EF, que resultaron similares al Estudio 1. Todos los CCIT-c presentaron valores $>.75$, excepto el ítem 13 (-.01). También en este caso, la correlación entre la puntuación de cada ítem y la puntuación total en cada uno de los componentes muestran correlaciones positivas y significativas con la dimensión teórica a la que pertenecen, excepto el ítem 13 que resultó muy baja $(r=.09)$.

Tabla 3

Estadísticos Descriptivos, de Consistencia Interna y de Homogeneidad $(N=758)$

\begin{tabular}{|c|c|c|c|c|c|c|c|}
\hline Escala: & $M$ & $S D$ & CCIT-c & $\mathrm{CC}$ & $\alpha$ sin ítem & Asimetría & Curtosis \\
\hline \multicolumn{8}{|c|}{ Apoyo a la autonomía $(\alpha=95)$} \\
\hline Ítem-2 & 4.77 & 1.93 & .85 & .87 & .94 & -.50 & -.87 \\
\hline Ítem-4 & 5.00 & 1.87 & .85 & .87 & .94 & -.60 & -.72 \\
\hline Ítem-8 & 4.97 & 1.92 & .84 & .86 & .94 & -.62 & -.77 \\
\hline Ítem-11 & 4.59 & 1.93 & .83 & .86 & .94 & -.36 & -.97 \\
\hline Ítem-9 & 4.92 & 1.85 & .83 & .85 & .94 & -.60 & -.66 \\
\hline Ítem-5 & 5.28 & 1.76 & .79 & .82 & .94 & -.78 & -.38 \\
\hline Ítem-10 & 4.42 & 1.92 & .82 & .84 & .94 & -.32 & -.99 \\
\hline Ítem-7 & 4.97 & 1.83 & .76 & .80 & .94 & -.55 & -.55 \\
\hline Ítem-3 & 4.99 & 1.87 & .82 & .84 & .94 & -.59 & -.74 \\
\hline Ítem-6 & 5.05 & 1.78 & .77 & .80 & .94 & -.58 & -.65 \\
\hline Ítem-12 & 4.54 & 1.98 & .79 & .82 & .94 & -.32 & -1.08 \\
\hline Ítem-14 & 4.43 & 1.87 & .79 & .82 & .94 & -.24 & -.94 \\
\hline Ítem-1 & 4.58 & 1.87 & .74 & .77 & .95 & -.37 & -.82 \\
\hline Ítem-15 & 3.95 & 2.17 & .71 & .75 & .95 & -.01 & -1.39 \\
\hline Ítem-13 & 3.07 & 2.14 & -.01 & .09 & .96 & .60 & -1.05 \\
\hline
\end{tabular}

Nota. M: media; SD: desviación típica; CCIT-c: coeficiente de correlación corregido ítem-total; CC: correlación de cada ítem con la otra dimensión.

\section{Análisis Factorial Confirmatorio}

Con el objeto estudiar las propiedades psicométricas de la dimensionalización original propuesta teóricamente por Williams y Deci (1996) se aplicaron modelos de ecuaciones estructurales. La estructura factorial del LCQ se evaluó con AFC. Este análisis se llevó a cabo utilizando el método de estimación weighted least squares (WLS) para variables ordinales del programa LISREL 8.80 (Jöreskog \& Sörbom, 1993). La matriz de correlaciones policóricas y la matriz de covarianzas asintóticas fueron utilizadas como input para el análisis de los datos. Se hipotetizó un modelo de medida de un solo factor. 
Como recomiendan diversos autores (Markland, 2007), para la evaluación de los modelos se calcularon varios índices de ajuste, combinando índices absolutos y relativos. Entre los absolutos, se utilizó el valor $p$, asociado con el estadístico chi-cuadrado $\left(\chi^{2}\right)$; la ratio entre $\chi^{2}$ y grados de libertad $\left(g l ; \chi^{2} / g l\right)$ es un heurístico que se utiliza para reducir la sensibilidad del $\chi^{2}$ al tamaño de la muestra. Las ratios $<2.0$ se consideran como indicadores de muy buen ajuste del modelo (Tabachnick \& Fidell, 2007), mientras que valores $<5.0$ son considerados aceptables $(\mathrm{Hu} \&$ Bentler, 1999). Se ha calculado el GFI (índice de bondad

\section{MODELO 15 ÍTEMS}

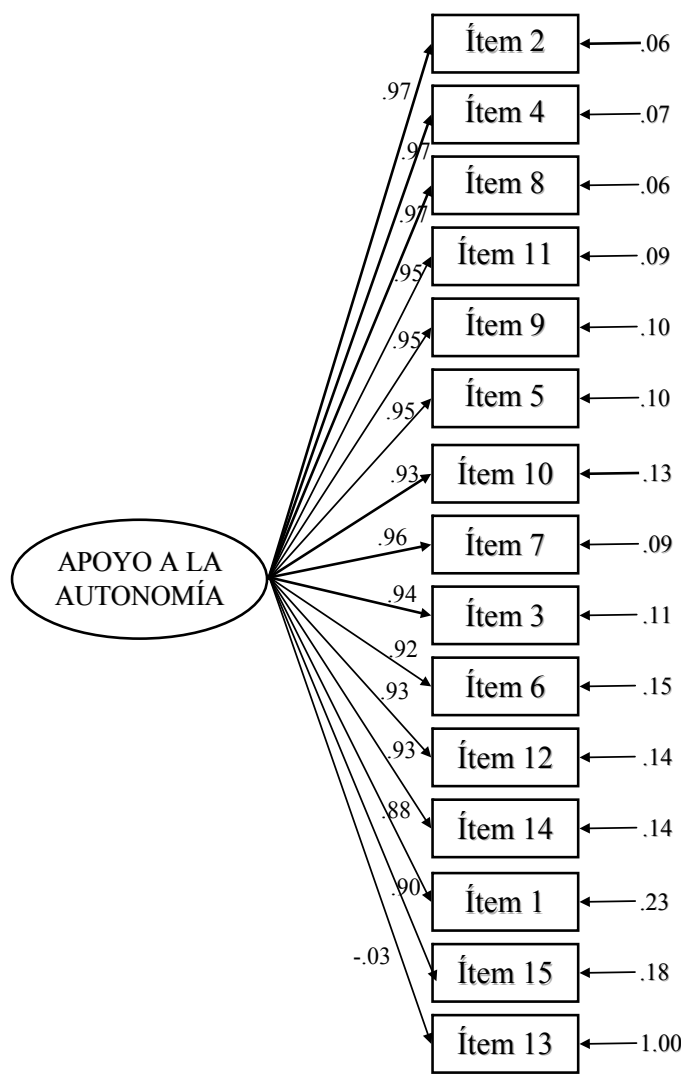

de ajuste) y autores como Hooper, Coughlan y Mullen (2008) consideran valores $\geq .95$ para un mejor ajuste. Entre los índices relativos se han utilizado: NFI (índice de ajuste normalizado), NNFI (índice de ajuste no normativo) y CFI (índice de ajuste comparativo). En los índices incrementales se considera que valores $\geq .95$ indican un buen ajuste (Hu \& Bentler, 1999). Autores como Kline (2005) recomiendan usar el RMSEA (error de aproximación cuadrático medio) y, según Hu y Bentler (1999), un valor $\leq .06$ indicaría un buen ajuste. Los parámetros estimados se consideran significativos cuando el valor asociado al valor $t$ es $>1.96(p<.05)$.

\section{MODELO 14 ÍTEMS}

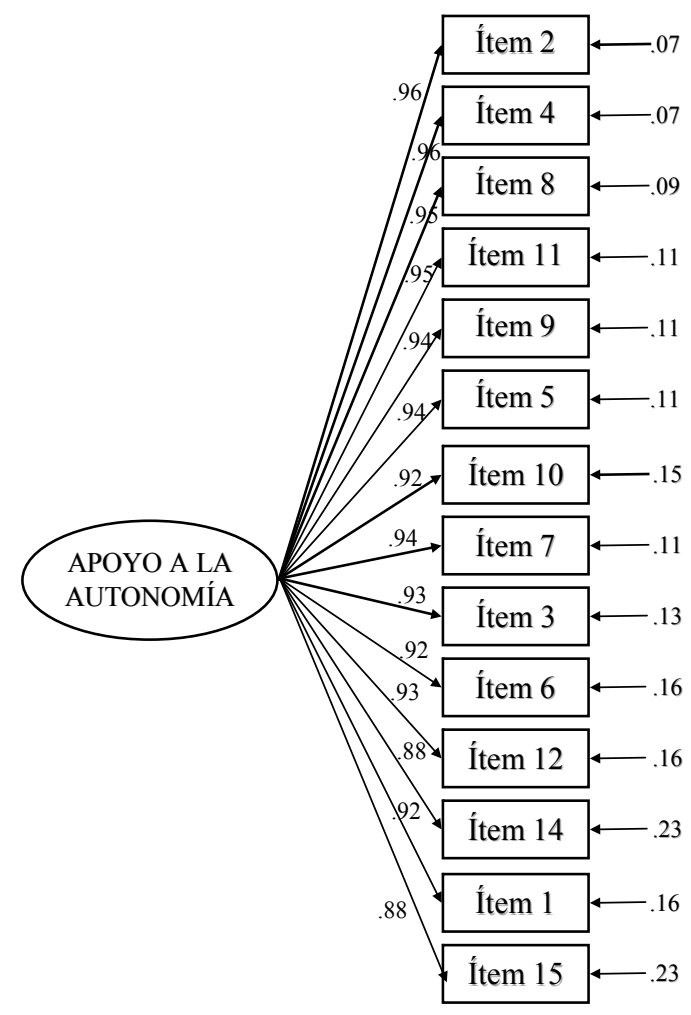

Figura 1. Path diagram del AFC, con pesos estandarizados y errores de medición de cada uno de los ítems del LCQ en alumnado de EF en secundaria.

Siguiendo recomendaciones de autores como Markland (2007), de formular y analizar varios modelos si los datos así lo recomiendan y de reportar los resultados más relevantes, seguidamente se presentan los resultados del AFC correspondientes a los dos modelos planteados (Figura 1): 15 y 14 ítems. El modelo con 14 ítems se efectuó sin el ítem-13. En el Path Diagram (Figura 1, izq.) se recoge el modelo con los valores del ítem 13, en el que destacan la baja carga factorial (-.03) y el alto error de medición (1.00). Todos los ítems presentaron valores $>.05$ en la fiabilidad individual $\left(R^{2}\right)$, excepto el ítem $13\left(R^{2}=.001\right)$, lo que recomienda su revisión y probar el modelo sin este ítem. En el modelo de 14 ítems, todos presentaron valores $>.05$ en $R^{2}$ (entre .75 del ítem 1 y .93 del ítem 4 ).

Se reportan los índices de ajuste de ambos modelos. El modelo de 15 ítems presentó un ajuste satisfactorio: $\chi^{2}=316.28, g l=90, p<.001, \chi^{2} / g l=3.52$, GFI $=.99$, NFI $=.98$, $\mathrm{NNFI}=.98, \mathrm{CFI}=.98, \mathrm{RMSEA}=.06$. En el modelo de 14 ítems los resultados mostraron un mejor ajuste: $\chi^{2}=201.75$, $g l=77, p<.001, \chi^{2} / g l=2.62, \mathrm{GFI}=.99, \mathrm{NFI}=.98, \mathrm{NNFI}=.98$, $\mathrm{CFI}=.99$, RMSEA $=.04$.

El modelo de 15 ítems no presenta los requisitos mínimos para poder garantizarla validez convergente del modelo (Hair, Black, Babin, \& Anderson, 2009): cargas 
Granero-Gallegos, A., Baena-Extremera, A., Sánchez-Fuentes, J. A. \& Martínez-Molina, M. (2014). Validación Española del "Learning Climate Questionaire" Adaptado a la Educación Física.

factoriales estandarizadas elevadas (en el ítem 13 no es $>.60$ ), aunque sí son todas estadísticamente significativas ( $t$-value $>1.96)$; ambas son condiciones necesarias para garantizar dicha validez. El modelo de 14 ítems sí permite garantizar la validez convergente de dicho modelo (Figura 1). En el Anexo se recoge la versión definitiva del LCQEF (14 ítems).

También es importante, en el AFC de las escalas con naturaleza ordinal de la matriz de correlaciones de datos, ofrecer los resultados de fiabilidad compuesta y varianza media extraída (AVE - Average Variante Extracted) para cada una de las dimensiones críticas. Según Hair et al. (2009) la fiabilidad compuesta ha tener un valor mínimo de .70 y la AVE de .50. En este trabajo, la AVE del LCQ$\mathrm{EF}=.86$ y la fiabilidad compuesta $=.99$.

A la vista de los resultados se evaluó la estabilidad temporal del instrumento con la citada muestra de 150 escolares. Los resultados del pretest en apoyo a la autonomía fueron: $\alpha=.96$; los del postest: $\alpha=.95$. Los valores de correlación test-retest: $r=.85$.

\section{Validez Concurrente}

La validez empírica se evaluó examinando la relación entre la puntuación total obtenida en el LCQ-EF y los tipos de motivación autodeterminada mediante SEM (WLS).

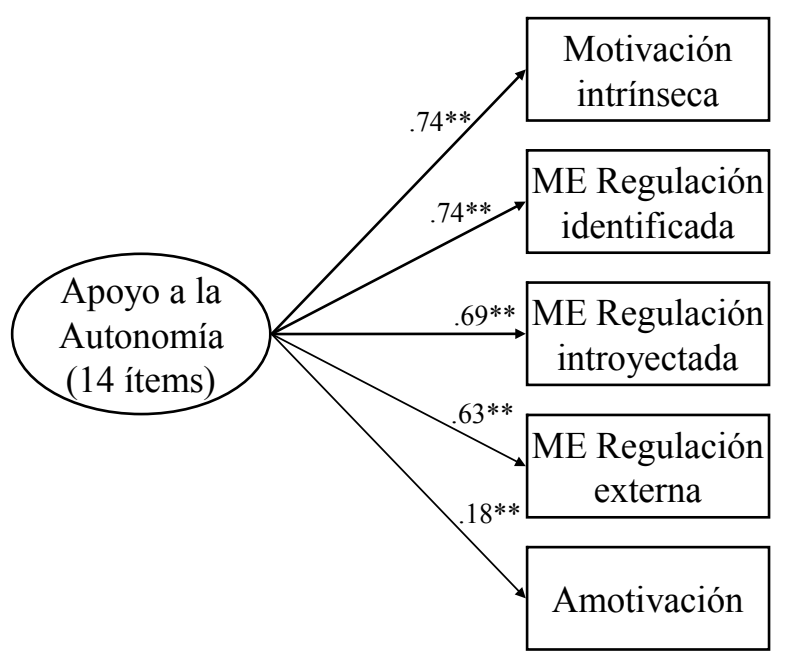

Figura 2. Solución estandarizada del modelo estructural hipotetizado (t-value $>1.96$ ) para la validez convergente (variables latentes).

El SEM del LCQ-EF (14 ítems) confirmó que el apoyo a la autonomía por parte del profesorado se mostró como el predictor más potente de las formas más autodeterminadas de motivación entre los estudiantes. Asimismo, aunque los valores son menores, también el apoyo a la autonomía es un predictor importante entre la ME menos autodeterminada. Los valores de predicción descienden considerablemente en relación con la amotivación (Figura 2). El modelo presentó índices de bondad de ajuste satisfac- torios: $\chi^{2}=2782.91, g l=814, p<.001, \chi^{2} / g l=3.41, \mathrm{GFI}=.96$, $\mathrm{NFI}=.97, \mathrm{NNFI}=.97, \mathrm{CFI}=.97, \mathrm{RMSEA}=.06$.

\section{Discusión}

El objetivo del presente trabajo consistió en examinar las propiedades psicométricas de la versión española del LCQ adaptado a la EF. La validez factorial de la escala LCQ fue investigada inicialmente por Williams y Deci (1996), obteniendo un autovalor de 9.5 en un único factor, con el $63 \%$ de varianza explicada. En este trabajo, el AFC basado en modelos de ecuaciones estructurales apoya la validez factorial y la fiabilidad del instrumento adaptado en una dimensión (apoyo a la autonomía), similar al del modelo hipotetizado de origen, y coincidente con los trabajos señalados.

A pesar de que el LCQ ha demostrado una buena consistencia interna, al obtenerse valores de alfa de Cronbach de .93, .94 (Black \& Deci, 2000) y .96 (Williams \& Deci, 1996; Williams et al., 1997), en esta investigación el ítem 13 no alcanza el coeficiente de visualización de .40 (Estudio 1). Además de los valores obtenidos de CCIT-C o su peso factorial, que invitan a su eliminación, el ítem 13 presenta una correlación negativa con la dimensión a la que teóricamente pertenece. Igualmente, en el AFC se aprecia una baja carga factorial y un alto error de medición. A pesar de ello, los datos obtenidos en el modelo de 15 ítems exponen un ajuste satisfactorio; no obstante, el modelo de 14 ítems mostró un mejor ajuste y asegura la validez convergente del modelo, mientras que el de 15 ítems no presenta los requisitos mínimos para poder garantizarla, según Hair et al. (2009). Por ello se propone la eliminación del ítem 13 de la escala; sugerencia que se encuentra en otros trabajos como los de Balaguer et al. (2009) y Yoshida y Nakano (2011). Además, se han utilizado otros indicadores, como el coeficiente de fiabilidad compuesta, la AVE o la estabilidad temporal; todos ellos dan muestra de la fiabilidad y validez de esta escala con 14 ítems. Asimismo, indicar que esta escala ha sido analizada factorialmente tanto desde el ámbito del deporte, (Balaguer et al., 2009; Reinboth, Duda, \& Ntoumanis, 2004; Williams et al., 1996) como de la EF (Hagger et al., 2003; Ntoumanis, 2005), obteniendo buenos índices de fiabilidad y ajuste.

En la validez empírica, el modelo de 14 ítems del LCQEF confirmó que el apoyo a la autonomía por parte del profesor y se mostró como un predictor, preferentemente, de las formas más autodeterminadas de motivación entre los estudiantes adolescentes, coincidiendo con las aportaciones de Black y Deci (2000), Hagger et al. (2003; Hagger et al., 2009), Standage, Gillison, Ntoumanis y Treasure (2012) y Taylor y Ntoumanis (2007). En sentido contrario, cuando el clima de clase en EF no apoya la autonomía, los niveles de motivación intrínseca bajan en los alumnos (Vallerand, 2000). Estos resultados coinciden con los de Lim y Wang (2009) en estudiantes de EF, y por Balaguer et al. (2009) y Pelletier, Fortier, Vallerand y Briere (2001) 
en deportistas. Igualmente, esta predicción está presente en valores menores con la motivación extrínseca y menos aún con la amotivación. Estos mismos datos se presentan en el trabajo de Balaguer et al. (2009), coincidiendo en la misma tendencia, pero en el deporte.

Para concluir, se puede afirmar que a pesar de que ambos modelos presentaron índices de bondad de ajuste satisfactorios, los resultados obtenidos por el modelo de 14 ítems mejoran los del modelo de 15 ítems, en un único factor hipotetizado según la teoría de origen. No obstante, sería necesario que otras investigaciones continúen evaluando estos modelos. Los resultados obtenidos avalan la utilización del LCQ-EF, ya que resultan perfectamente coherentes con las escasas investigaciones existentes tanto en el ámbito académico como deportivo. Queda así demostrado que la LCQ-EF es un instrumento válido y fiable para evaluar el clima de apoyo a la autonomía que perciben los alumnos de EF por sus profesores en su versión española. Es de destacar la importancia que tiene esta escala y su gran utilidad para los docentes, tanto en la creación de clima de aprendizaje como en la creación de climas motivacionales, y la repercusión de éstos en los alumnos.

\section{Referencias}

Baena-Extremera, A., Granero-Gallegos, A., Sánchez-Fuentes, J. A., \& Martínez-Molina, M. (2013). Apoyo a la autonomía en Educación Física: antecedentes, diseño, metodología y análisis de la relación con la motivación en estudiantes adolescentes. Retos. Nuevas tendencias en Educación Física, Deporte y Recreación, 24, 46-49. Recuperado de http://www.retos.org/ numero 24/46-49.pdf

Balaguer, I., Castillo, I., Duda, J. L., \& Tomás, I, (2009). Análisis de las propiedades psicométricas de la versión española del Cuestionario de Clima en el Deporte. Revista de Psicología del Deporte, 18(1), 73-83. Recuperado de http://redalyc.uaemex. $\mathrm{mx} / \mathrm{redalyc} / \mathrm{pdf} / 2351 / 235119250005 . \mathrm{pdf}$

Black, A. E., \& Deci, E. L. (2000). The effects of instructors' autonomy support and students' autonomous motivation on learning organic chemistry: A self-determination theory perspective. Science Education, 84, 740-756. doi:10.1002/1098237X(200011)84:6<740::AID-SCE4>3.0.CO;2-3

Boekaerts, M. (1996). Self-regulated learning at the junction of cognition and motivation. European Psychologist, 1(2), 100-112. doi:10.1027/1016-9040.1.2.100

Brislin, R. W. (1986). The wording and translation of research instruments. In W. Lonnery \& J. Berry (Eds.), Field methods in cross-cultural research (pp. 137-164). Beverly Hills, CA: Sage.

Carretero-Dios, H., \& Pérez, C. (2007). Standards for the development and the review of instrumental studies: Considerations about test selection in psychological research. International Journal of Clinical and Health Psychology, 7, 863-882. Retrieved from http://www.aepc.es/ijchp/articulos_pdf/ ijchp-257.pdf

Deci, E. L., \& Ryan, R. M. (1985). Intrinsic motivation and self-determination in human behavior. New York: Plenum.

Hagger, M. S., Chatzisarantis, N., Culverhouse, T., \& Biddle, S. J. H. (2003). The processes by which perceived autonomy support in physical education promotes leisure-time physical activity intentions and behavior: A trans-contextual model. Journal of Educational Psychology, 95, 784-795. doi:10.1037/0022-0663.95.4.784

Hagger, M. S., Chatzisarantis, N. L., Hein, V., Pihu, M., Soos, I., Karsai, I., ...Leemans, S. (2009). Teacher, peer, and parent autonomy support in physical education and leisuretime physical activity: A trans-contextual model of motivation in four nations. Psychology and Health, 24, 689-711. doi:10.1080/08870440801956192

Hair, J. F., Black, W. C., Babin, B. J., \& Anderson, R. E. (2009). Multivariate data analysis ( $7^{\text {th }}$ ed.). New York: Pearson Prentice Hall.

Hooper, D., Coughlan, J., \& Mullen, M. (2008). Structural equation modelling: Guidelines for determining model fit. Electronic Journal of Business Research Methods, 6(1), 5360. Retrieved from http://www.ejbrm.com/volume6/issue1

Hu, L., \& Bentler, P. M. (1999). Cutoff criteria for fit indexes in covariance structure analysis: Conventional criteria versus new alternatives. Structural Equation Modelling, 6, 1-55. doi:10.1080/10705519909540118

Jöreskog, K. G., \& Sörbom, D. (1993). Structural equation modelling with the SIMPLIS command language. Chicago, IL: Scientific Software International.

Kline, R. B. (2005), Principles and practice of structural equation modelling ( $2^{\text {nd }}$ ed.). New York: The Guilford Press.

Lim, B., \& Wang, C. (2009). Perceived autonomy support, behavioural regulations in physical education and physical activity intention. Psychology of Sport and Exercise 10, 5260. doi:10.1016/j.psychsport.2008.06.003

Markland, D. (2007). The golden rule is that there are no golden rules: A commentary on Paul Barrett's recommendations for reporting model fit in structural equation modelling. Personality and Individual Differences, 42(5), 851-858. doi:10.1016/j. paid.2006.09.023

Matos, L. (2009). Adaptación de dos cuestionarios de motivación: autorregulación del aprendizaje y clima de aprendizaje. Persona, 12, 167-185. Recuperado de http://redalyc.uaemex.mx/ src/inicio/ArtPdfRed.jsp?iCve $=147117618010$

Muñiz, J. (2000). Adaptación de los test de unas culturas a otras. Metodología de las Ciencias del Comportamiento, 2(2), 129-149.

Muñiz, J., \& Bartram, D. (2007). Improving international tests and testing. European Psychologist, 12, 206-219. doi:10.1027/1016-9040.12.3.206

Ntoumanis, N. (2001). A self-determination approach to the understanding of motivation in physical education. British Journal of Educational Psychology, 71(2), 225-242. doi:10.1348/000709901158497

Ntoumanis, N. (2005). A prospective study of participation in optional school physical education using a self-determination theory framework. Journal of Educational Psychology, 97, 444-453. doi:10.1037/0022-0663.97.3.444

Núñez, J. L., Martín-Albo, J., Navarro, J. G., \& González, V. M. (2006). Preliminary validation of a Spanish version of the Sport Motivation Scale. Perceptual and Motor Skills, 102, 919-930. doi:10.2466/pms.102.3.919-930

Nunnally, J. C., \& Bernstein, I. J. (1995). Teoría psicométrica. Madrid, España: McGraw-Hill.

Osterlind, S. J. (1989). Constructing test items. London: Kluwer Academic.

Pelletier, L. G., Fortier, M. S., Vallerand, R. J., \& Briere, N. M. (2001). Associations among perceived autonomy support, forms of self-regulation, and persistence: A prospective study. Motivation and Emotion, 25, 279-306. doi:10.1023/A:1014805132406 
Reeve, J., Jang, H., Carrell, D., Jeon, S., \& Barsh, J. (2004). 'Enhancing students' engagement by increasing teachers 'autonomy support'. Motivation and Emotion, 28, 147-169. doi:10.1023/B:MOEM.0000032312.95499.6f

Reinboth, M., Duda, J. L., \& Ntoumanis, N. (2004). Dimensions of coaching behavior need satisfaction, and the psychological and physical welfare of young athletes. Motivation and Emotion, 28(3), 297-313. doi:10.1023/ B:MOEM.0000040156.81924.b8

Ryan, R. M., \& Deci, E. L. (2000). Self-determination theory and the facilitation of intrinsic motivation, social development and well-being. American Psychologist, 55(1), 68-78. doi:10.1037/0003-066X.55.1.68

Standage, M., Duda, J. L., \& Ntoumanis, N. (2005). A test of self-determination theory in school physical education. British Journal of Educational Psychology, 75(3), 411-433. doi:10.1348/000709904X22359

Standage, M., Gillison, F. B., Ntoumanis, N., \& Treasure, D. (2012). Predicting students' physical activity and healthrelated well-being: A prospective cross-domain investigation of motivation across school physical education and exercise settings. Journal of Sport \& Exercise Psychology, 34, 37-60. Retrieved from http://www.selfdeterminationtheory.org/SDT/ documents/2012_StandageGillisonNtoumanisTreasure_JSEP. pdf

Stevens, J. (1992). Applied multivariate statistics for the social sciences. Hillsdale, NJ: Lawrence Erlbaum.

Tabachnick, B. G., \& Fidell, L. S. (2007). Using multivariate statistics $\left(5^{\text {th }}\right.$ ed.). New York: Allyn and Bacon.

Taylor, I. M., \& Ntoumanis, N. (2007). Teacher motivational strategies and student self-determination in physical education. Journal of Educational Psychology, 99(2), 747-760. doi:10.1037/0022-0663.99.4.747
Vallerand, R. J. (2000). Deci and Ryan's self-determination theory: A view from the hierarchical model of intrinsic and extrinsic motivation. Psychological Inquiry, 11(4), 312-318.

Williams, G. C., \& Deci, E. L. (1996). Internalization of biopsychosocial values by medical students: A Test of SelfDetermination Theory. Journal of Personality and Social Psychology, 70(4), 767-779.

Williams, G. C., Grow, V. M., Freedman, Z. R., Ryan, R. M., \& Deci, E. L. (1996). Motivational predictors of weight loss and weight-loss maintenance. Journal of Personality and Social Psychology, 70(1), 115-126.

Williams, G. C., Saizow, R., Ross, L., \& Deci, E. L. (1997). Motivation underlying career choice for internal medicine and surgery. Social Science and Medicine, 45(11), 1705-1713. doi:10.1016/S0277-9536(97)00103-2

Yoshida, S., \& Nakano, M. (2011, August). A pilot study on the relationship between learning climate in English classroom and students' motivation toward the classroom activities. In Proceedings of the $16^{\text {th }}$ Conference of Pan-Pacific Association of Applied Linguistics (pp. 357-364). Sha Tin, Hong Kong: The Chinese University of Hong Kong. Retrieved from http://paaljapan.org/conference2011/ProcNewest2011/pdf/ poster/Q-8.pdf

1. Siento que mi profesor/a de EF me ofrece distintas alternativas y opciones

2. Me siento comprendido/a por mi profesor/a de EF

3. Tengo la posibilidad de poder comunicarme fácilmente con mi profesor/a durante la clase de EF

4. Mi profesor/a de EF me transmite confianza en mi habilidad para hacerlo bien en las clases

5. Me siento aceptado/a por mi profesor/a de EF

6. Mi profesor/a de EF se asegura que yo realmente entienda cuál es el objetivo de cada actividad de

clase y de lo que tengo que hacer

7. Mi profesor/a de EF me anima a que pregunte en clase las dudas que tenga

8. Confío mucho en mi profesor/a de EF

9. Mi profesor/a de EF responde a mis preguntas con esmero (detenidamente y en detalle)

10. A mi profesor/a de EF le interesa saber como me gusta hacer las cosas

11. Mi profesor/a de EF maneja muy bien las emociones de la gente

12. Siento que mi profesor/a de EF se preocupa de mí como persona

13. Mi profesor/a de EF trata de entender como veo las cosas antes de sugerirme una nueva manera de hacerlas

14. Me siento capaz de compartir mis sentimientos con mi profesor/a de EF 